\title{
Characteristics of patients hospitalized for heart failure at the University Hospital Centre Split stratified by left ventricular ejection fraction
}

\author{
(iD)Anita Jukićc ${ }^{*}$, \\ (D) Katarina Kutleša², \\ (DDamir Fabijanićn ${ }^{1,2}$ \\ University Hospital Centre \\ Split, Split, Croatia \\ 2University of Split School of \\ Medicine, Split, Croatia
}

\begin{abstract}
KEYWORDS: heart failure, ejection fraction, clinical characteristics, comorbidities. CITATION: Cardiol Croat. 2021;16(1-2):24. | https://doi.org/10.15836/ccar2021.24
\end{abstract}

*ADDRESS FOR CORRESPONDENCE: Anita Jukić, Klinički bolnički centar Split, Spinčićeva 1, HR-21000 Split, Croatia. / Phone: +385-95-9072444 / E-mail: anitajkc@gmail.com

ORCID: Anita Jukić, https://orcid.org/0000-0002-1694-9216 • Katarina Kutleša, https://orcid.org/0000-0002-4267-2169 Damir Fabijanić, https://orcid.org/0000-0002-4199-3905

|IIIIIIIIIIIIIIIIIIIIIIIIIIIIIIIIIIIIIIIIIIIIIIIIIIIIIIIIIIIIIIIIIIIIIIIIIIIIIIIIIIIIIIIIIIIIIIIIIIIIIIIIIIIIIIIIIIIII

Introduction: The objective of this study was to analyze characteristics of the patients hospitalized because of heart failure according to their left ventricular ejection fraction (EF).

Patients and Methods: We retrospectively analyzed data on $293 \mathrm{HF}$ patients hospitalized at the Department of Cardiology of the University Hospital of Split between January 1st and December 31st, 2019. The patients were divided according to left ventricular ejection fraction (EF) into 3 categories: 1) HF with preserved EF (HFpEF), 2) HF with midrange EF (HFmrEF) and 3) HF with reduced EF (HFrEF). Categorical variables were described as absolute or relative prevalence and assessed by $\mathrm{x}^{2}$ test; analysis of variance (ANOVA) was used to compare normally distributed quantitative variables; Kruskal-Wallis ANOVA was used to compare non-normally distributed quantitative variables.

Results: Among 293 HF patients, 125 (43\%) had HFrEF, 61 (21\%) had HFmrEF and 107 (36\%) had HFpEF. Patients with HFrEF were mostly men (72\%), younger than 70 years (62\%), most commonly with arterial hypertension (54.4\%) and revascularized coronary artery disease (36\%). Patient with HFmrEF were mostly men (59\%), older than 70 years (60.66\%), with arterial hypertension (80.33\%), permanent atrial fibrillation (63.93\%) and revascularized coronary artery disease (31\%). Patients with HFpEF were mainly women (61.68\%), older than 70 years (61.68\%), with arterial hypertension (80.37\%), atrial fibrillation (60.75\%), valvular heart diseases (25.23\%) and chronic obstructive pulmonary disease (12,15\%).

Conclusion: The largest number of hospitalized patients diagnosed with HF had a reduced EF. These patients were more likely to be younger men with previously revascularized coronary artery disease, whereas HFpEF patients were predominantly elderly females with arterial hypertension and permanent atrial fibrillation. Despite the fact that they were mostly men, according to clinical characteristics, the patients with HFmrEF were significantly more similar to patients with HFpEF. ${ }^{1}$

\section{RECEIVED:}

December 6, 2020

ACCEPTED:

December 18, 2020

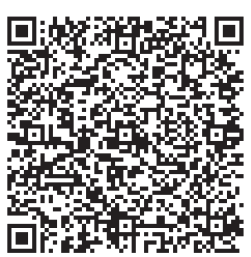

$\square$ Cardiologia Croatica 2021;16(1-2):24.
LITERATURE IIIIIIIIIIIIIIIIIIIIIIIIIIIIIIIIIIIIIIIIIIIIIIIIIIIIIIIIIIIIIIIIIIIIIIIIIIIIIIIIIIIIIIIIIIIIIIIII

1. Ponikowski P, Voors AA, Anker SD, Bueno H, Cleland JG, Coats AJ, et al; Authors/Task Force Members; Document Reviewers. 2016 ESC Guidelines for the diagnosis and treatment of acute and chronic heart failure: The Task Force for the diagnosis and treatment of acute and chronic heart failure of the European Society of Cardiology (ESC). Developed with the special contribution of the Heart Failure Association (HFA) of the ESC. Eur J Heart Fail. 2016 Aug;18(8):891-975. https://doi.org/10.1002/ejhf.592 